\title{
Advances in the Development of White-Light Interferometry for In-Situ Uranium Hydride Kinetic Data Collection
}

Yaakov Idell $^{1}$, Wigbert Siekhaus ${ }^{2}$, Kerri Blobaum ${ }^{2}$ and William McLean ${ }^{2}$

${ }^{1}$ Lawrence Livermore National Laboratory, San Jose, California, United States, ${ }^{2}$ Lawrence Livermore National Laboratory, United States

Uranium hydriding is a corrosion process that causes significant material degradation and poses a serious concern for long-term storage and disposal. Hydrogen can locally attack the uranium to form uranium hydride $\left(\mathrm{UH}_{3}\right)$ when uranium is exposed to a hydrogen-rich, or water-based environment interacts with insoluble hydrogen present in the matrix through impurities in the fabrication process. Hydrogen has been shown to increase brittleness of the uranium and to produce unfavorable properties of tensile strength, hardness, and elongation [1].

Because the $\mathrm{UH}_{3}$ density $\left(10.92 \mathrm{~g} / \mathrm{cm}^{3}\right)$ [2] is lower than that of the parent uranium metal $\left(19.05 \mathrm{~g} / \mathrm{cm}^{3}\right)$ [2], the transformation of uranium into $\mathrm{UH}_{3}$ will cause considerable local expansion (volumetric increase of $\approx 60 \%$ ). If this transformation occurs in a location constrained by the uranium substrate (i.e., at an inclusion-uranium interface), local deformation and potentially fracture may occur due to volumetric strain if the $\mathrm{UH}_{3}$ precipitate grows above a critical size. Corrosion of uranium by hydrogen has typically been characterized by an initiation time followed by pitting corrosion; however, variability in the $\mathrm{UH}_{3}$ initiation time and thickness of the coherent uranium oxide $\left(\mathrm{UO}_{2}\right)$ film have made understanding of earlystage $\mathrm{UH}_{3}$ growth quite difficult. Previous studies to understand $\mathrm{UO}_{2}$ or $\mathrm{UH}_{3}$ growth kinetics and mechanisms have employed characterization tools such as a microbalance [3], X-ray diffraction [4], spectroscopic ellipsometry [5], Fourier-transform infrared spectroscopy [6], and hot-stage optical microscopy [7]. These techniques provide quality data but provide unsatisfactory data in early-stage growth studies because their detection limits are not sufficiently sensitive to the initial thin layers or with the necessary time-resolution. Drawbacks of these techniques include weak signal due to material absorption, indirect measurements requiring modelling of data that can lead to error during interpretation, long sampling times, and spatial resolution that does not adequately capture early-stage growth data. We will introduce a novel characterization tool that can rapidly collect time-dependent in-situ spatial data using while-light interferometry that can quantify early-stage hydride growth.

White-light interferometry is a non-destructive and non-contact optical surface profiling technique for materials characterization that offers excellent lateral and vertical resolution. This optical profiling technique is based on interpreting a time-series of interferograms, a pattern of dark and bright lines, resulting from constructive and destructive interference caused by the optical path length difference between a beam interacting with a reference material and the sample of interest. The interferograms are individually processed using a frequency-domain analysis algorithm, which converts the vertical measurements interferometrically for each pixel in field of view creating a three-dimensional image of the sample surface. Figure 1 shows a schematic of a white-light interferometer.

The white-light interferometer is equipped with a computer-controlled $x-y$ stage that carries a customdesigned environmental sample cell permitting exposure to hydrogen gas pressures (dynamic or static) and heating for the in-situ experiments. Recent automation improvements for data collection have 
significantly increased the total field of view, which allows the ability to collect statistically representative data of the $\mathrm{UH}_{3}$ early-stage growth kinetics. In conventional microscopy, growth of the $\mathrm{UH}_{3}$ is typically characterized once the surface layer has erupted due to localized strain caused by the $\mathrm{UH}_{3}$. In contrast, white-light interferometry data can be collected related to the blistering of the surface layer caused by a sub-surface $\mathrm{UH}_{3}$ particle growth prior to surface eruption, which is important to accurate modeling and understanding the growth mechanisms. Figure 2 shows an example of $\mathrm{UH}_{3}$ growth of a single particle as a function of time where the black pixels indicate the non-reflective $\mathrm{UH}_{3}$. Black pixels on a white-light interferometry map indicate areas of "no data", which can be the result of be the result of the surface not having enough surface reflectivity or a surface protrusion with an angle that is too steep for sufficient surface reflection. In figure 2, the black pixels are attributed to the non-reflective hydride formation. The "no data" signal confirms that the black pixels are indeed from the hydride formation because of the collected height data from the preliminary blister prior to eruption. Results of experiments at different temperatures will be presented describing of the $\mathrm{UH}_{3}$ growth kinetics and morphology for both sub-surface blistering and post-surface eruption. Additionally, the data collection, depending on the material, can produce multiple sets of interferograms from multiple additional film layers present (i.e., potentially, an additional interferogram caused by an oxide layer can be present). Even though our experiments did not experience any issues with multiple sets of interferograms, strategies to address and resolve this phenomenon in order to attain the highest quality results will be discussed.

Lawrence Livermore National Laboratory is operated by Lawrence Livermore National Security, LLC, for the U.S. Department of Energy, National Nuclear Security Administration under Contract DE-AC5207NA27344. 


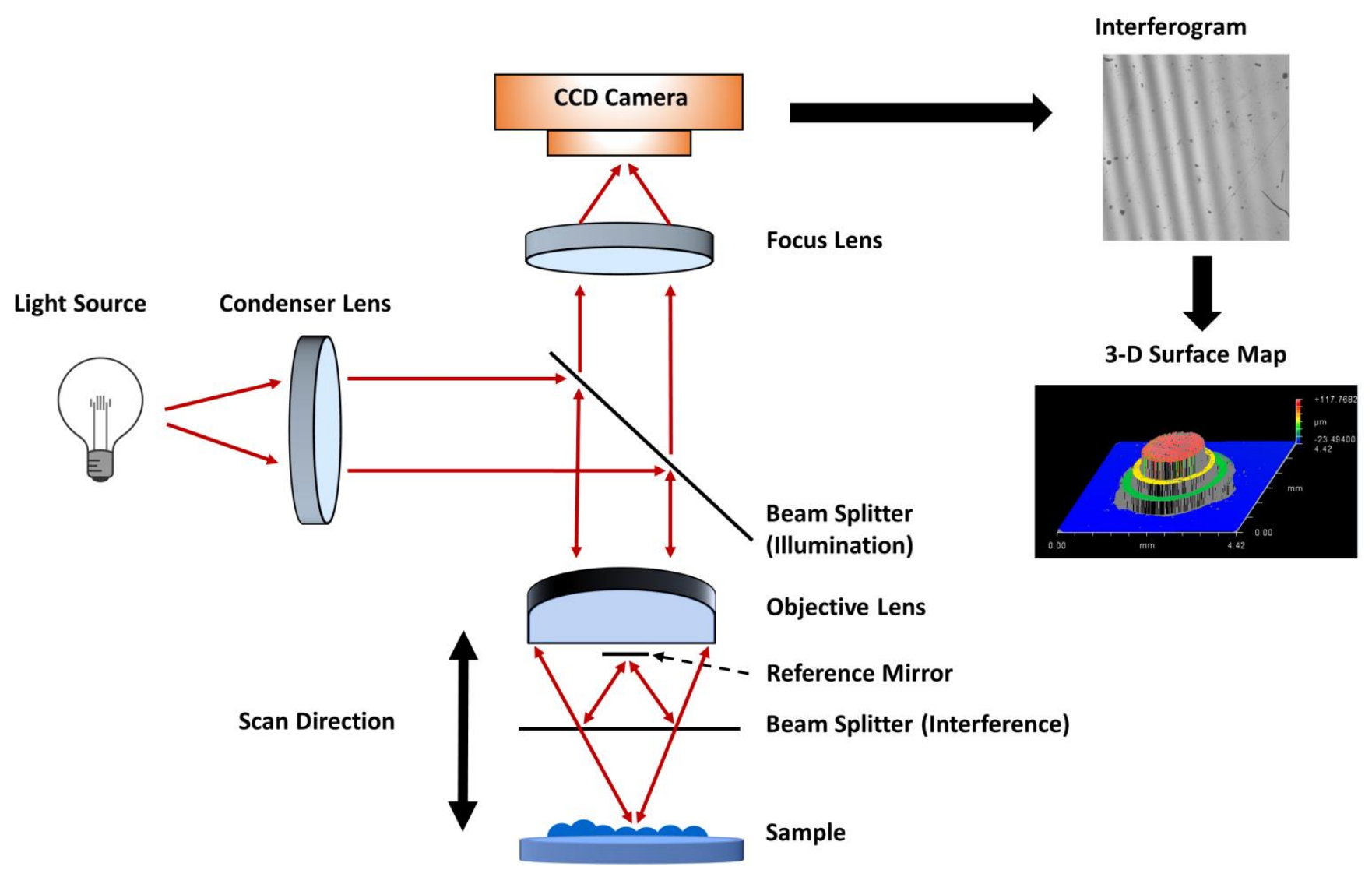

Figure 1. Figure 1: Schematic of a white-light interferometer
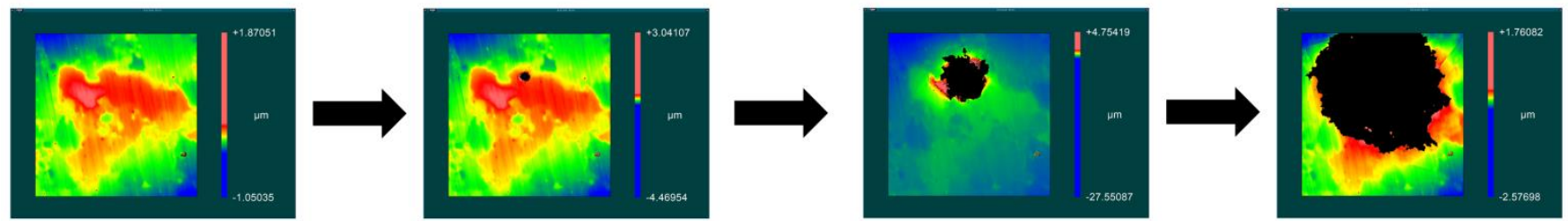

Figure 2. Figure 2: Growth of a single UH3 particle (black central spot) can be observed as a function of time.

\section{References}

[1] L. Marsh, G.Muehlenkamp, and G. Manning, "Effect of Hydrogen on the Tensile Transition in Uranium”, BMI (1955) 980.

[2] J.J. Katz and E. Rabinowitch, "The Chemistry of Uranium: The Element, Its Binary and Related Compounds" (Dover, New York, 1961).

[3] M. Bennett, B. Myatt, D. Silvester, and J. Antill, "The oxidation behavior of uranium in at 50-300" C", J. Nucl. Mater. 57 (1975) 221-236.

[4] S. Zalkind, G. Rafailov, I. Halevy, T. Livneh, A. Rubin, H. Maimon, and D. Schweke, "Uranium oxidation kinetics monitored by in-situ X-ray diffraction”, J. Nucl. Mater. 485 (2017) 202-206.

[5] R.E. Sladky, "Ellipsometric Study of the Corrosion of Uranium and Uranium-7.5 Weight Percent Niobium-2.5 Weight Percent Zirconium Alloy", Union Carbide Corp., Oak Ridge, Tenn. Y-12 Plant (1972). 
[6] D.B. Mawhinney, J.A. Glass, and J.T. Yates, "FTIR study of the oxidation of porous silicon", J. Phys. Chem. B. 101 (1997) 1202-1206.

[7] J. Bloch, F. Simca, M. Kroup, A. Stern, D. Shmariahu, M. Mintz, and Z. Hadari, "The initial kinetics of uranium hydride formation studied by a hot-stage microscope technique", J. Less Common Met. 103 (1984) 163-171. 